MS05-P08

\section{Mechanistic study of human phosphoserine phosphatase (hPSP) via crystallization with intermediates}

\author{
Marie Haufroid ${ }^{1}{ }^{2}$, Manon Mirgaux ${ }^{1}{ }^{2}$, Johan Wouters ${ }_{-}^{1}{ }^{2}$ \\ 1. Laboratoire de Chimie Biologique Structurale (CBS), Université \\ de Namur (UNamur) \\ 2. Namur Medicine \& Drug Innovation Center (NAMEDIC- \\ NARILIS) Namur, Belgium \\ email: marie.haufroid@unamur.be
}

Human phosphoserine phosphatase (hPSP) catalyses the third and final step of the serine pathway, which is the transformation of O-Phospho-L-Serine into L-Serine. This $\mathrm{Mg}^{2+}$ enzyme is part of the Haloacid Dehalogenase superfamily of enzymes and possesses a Rossman-like domain in order to orient the substrate within the active site. This protein is also a therapeutic target in colorectal cancer [1]

In this work, hPSP was produced, purified and crystallized in various conditions. In order to design inhibitors and gain a better understanding of the catalytic mechanism, we co-crystallized the enzyme in presence of different substrates and inhibitors. Crystals were obtained and data were collected at SOLEIL Synchrotron in France.

After resolution, we obtained a co-crystal of hPSP with phosphate as a ligand at a resolution of $1.5 \AA$. We also resolved a dynamic loop that was absent in the other high-resolution structure of hPSP (PDB: 1NNL) [2]. This loop was also described by Kim et al with a resolution range going from 2.5 to $2.8 \AA$ but was placed in a different manner (PDB: 1L8L \& 1L8O) [3]. The dynamic properties of this chain indicates a possible induced fit in order to open and close the active site during reaction.

We will complete those results thanks to other co-crystallization assays of hPSP with ligands. Moreover, we will apply dynamic simulations to elucidate the catalytic mechanism of this enzyme.

Acknowledgment. The authors thank members of namedic who generated namedic library used for inhibition and crystallization assays, in particular Prof B. Masereel and L. Pochet. Authors also acknowledge SOLEIL for provision of synchrotron radiation facilities and we would like to thank Leonard Chavas for assistance in using beamline Proxima I.

References:

[1] Li, X. et al (2016). Biochemical and Biophysical Research Communications, 477, 633-639

[2] Peeraer, Y. et al (2003). Acta Crystallographica Section D Biological Crystallography, D59, 971-977

[3] Kim, H-Y. et al (2002). Journal of Biological Chemistry, 277, 46651-46658.

Keywords: Phosphoserine phosphatase, protein crystallization, co-crystallization

\section{MS05-P09}

\section{Structural investigation of the interaction between Combretastatin A-4 analogues with benzoxazolone scaffold and $\alpha \beta-$ tubulin}

Rusi Rusev ${ }^{1}$, Mariana Gerova ${ }^{2}$, Georgi Atanasov ${ }^{3}$, Desislava

Stanisheva $^{2}$, Hristina Sbirkova-Dimitrova ${ }^{4}$, Margarita Apostolova ${ }^{3}$, Ognyan Petrov ${ }^{2}$, Boris Shivachev ${ }^{4}$

1. Institute of mineralogy and crystallography -Bulgarian academy of sciences, Sofia, Bulgaria

2. Faculty of chemistry and pharmacy, Sofia University, Sofia, Bulgaria

3. Institute of Molecular Biology "Acad. R. Tsanev", Bulgarian Academy of Sciences, Sofia, Bulgaria

4. Institute of Mineralogy and Crystallography "Acad. Iv. Kostov", Bulgarian Academy of Sciences, Sofia, Bulgaria

email: r.rusev93@gmail.com

A series of 70 new Combretastatin A-4 (CA4) analogues have been synthesized by modified Wittig reaction [1]. The compounds were tested in vitro for cell growth inhibition and the ability to induce apoptosis against HepG2, EA.hy926 and K562 cell line. The most active compounds were further tested against HT-29, Colon-26, A-549, MCF-7, MDA-MD-231, MCF-10A, HaCaT and NHEK lines. The new compounds have been characterized by single crystal diffraction and preliminary data about the interaction of the most active CA-4 analogues has been obtained using fluorescence displacement assay. The crystallization of selected analogues with $\alpha \beta$-tubulin-RSB complex suggested that interaction might occur at different sites though the "small molecules" are structurally very alike (Fig. 1). Figure 1. General scheme of synthesized analogues a), ORTEP view of the molecule of compound SZ16 present in the asymmetric unit [1] b) and possible interaction (binding) sites of the analogues with $\alpha \beta$-tubulin dimmer.

Acknowledgments: The authors would like to thank the Bulgarian National Science Found project H19/13 2017.

a)

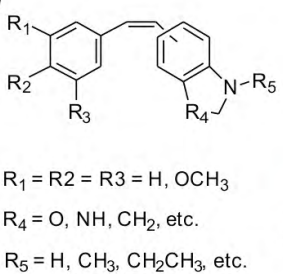

b)

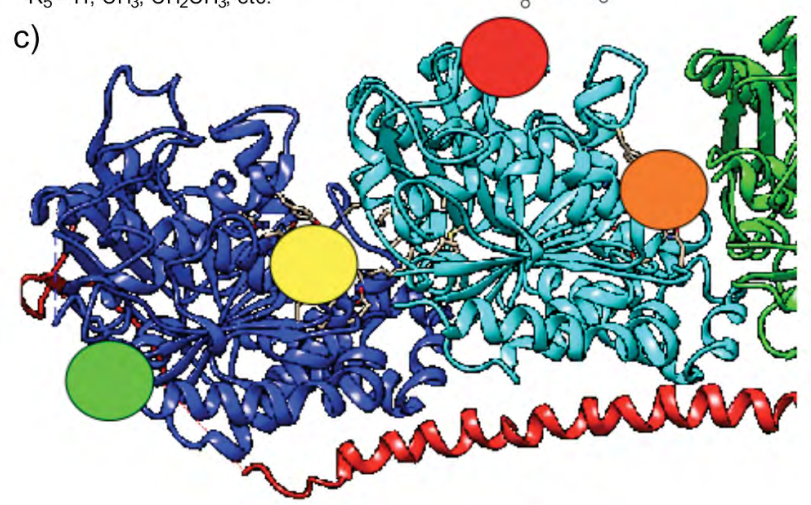

References:

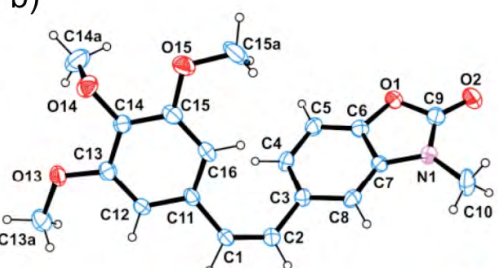

Gerova, M. S., et al. (2016) Eur. J. Med. Chem. 120, 121-133.

Keywords: Stilbene, Tubulin binding, Anticancer agents 